\title{
Expectativas de estudiantes sobre su formación universitaria. Carrera de Obstetricia. Instituto Dr. Andrés Barbero. San Lorenzo-Paraguay. 2018
}

\author{
Fátima Beatriz Sparling Pereira ${ }^{1}$ (D), Elena María Rodríguez Oliveira1 ${ }^{1 D}$ \\ ${ }^{1}$ Universidad Nacional de Asunción, Facultad de Enfermería y Obstetricia, Coordinación de \\ Investigación, Carrera de Obstetricia, Casa Central. San Lorenzo-Paraguay
}

Cómo referenciar este artículo/ How to reference this article:
Sparling Pereira FB, Rodríguez Oliveira EM. Expectativas de estudiantes sobre su formación universitaria. Carrera de Obstetricia. Instituto Dr. Andrés Barbero. San Lorenzo-Paraguay. 2018. Mem. Inst. Investig. Cienc. Salud. 2021; 19(1): $79-85$

\begin{abstract}
RE S U M E N
Desde el ingreso a la universidad cada estudiante tiene ideas y creencias en cuanto a su formación académica, con respecto a la elección de la carrera, a la institución y especialmente sobre las expectativas en cuanto a su futuro profesional, donde la calidad de su formación prevalecerá para la inserción laboral. El objetivo general fue determinar expectativas del estudiante sobre su formación universitaria en la carrera de Obstetricia del Instituto Dr. Andrés Barbero en San Lorenzo-Paraguay en 2018. El estudio tuvo un diseño observacional, descriptivo, corte transversal enfoque cuantitativo. La población original era de 253 estudiantes, pero la muestra fueron todos los estudiantes matriculados (25) del primer curso. El método usado fue la entrevista, la técnica una encuesta, y el instrumento un cuestionario impreso. Con el visto bueno de la máxima autoridad, se contactó con los sujetos de estudio, a fin de realizar la recolección de la información. Los datos fueron cargados en Excel, y analizados con EPIINFO 7.2 usando estadística descriptica. Casi la totalidad correspondió a mujeres del 17 a 19 años, poco más de la mitad era de procedencia urbana, y menos de la mitad tenía una familia nuclear. En cuanto a la motivación poco menos de la mitad ingresó al Instituto Dr. Andrés Barbero por razones personales, optó por la carrera de Obstetricia por motivos profesionales y en cuanto a las expectativas de los estudiantes sobre su formación universitaria predominaron las razones personales.
\end{abstract}

Palabras clave: Expectativas, estudiantes, formación universitaria.

\section{Student expectations about their university education. Obstetrics career. Instituto "Dr. Andrés Barbero". San Lorenzo-Paraguay. 2018}

ABSTRACT

Since entering the university, each student has ideas and beliefs regarding their academic training, regarding the choice of career, the institution and especially about the expectations regarding their professional future, where the quality of their training will prevail. for job placement. The general objective was to determine the student's expectations about their university training in the Obstetrics career of the Instituto "Dr. Andrés Barbero" in San Lorenzo-Paraguay in 2018. The study had an observational, descriptive, cross-sectional and quantitative approach design. The original population was 253 students, but the sample was all students enrolled (25) in the first year. The method used was the interview, the technique a survey, and the instrument a printed questionnaire. With the approval of the highest authority, the study subjects were contacted in order to collect the information. The data were Fecha de recepción: Agosto 2020. Fecha de aceptación: Marzo 2021

*Autor correspondiente: Fátima Beatriz Sparling Pereira. Universidad Nacional de Asunción, Facultad de Enfermería y Obstetricia, Coordinación de Investigación, Carrera de Obstetricia, Casa Central. San Lorenzo-Paraguay Email: sparling404@gmail.com 
loaded into Excel, and analyzed with EPIINFO 7.2 using descriptive statistics. Almost all of them corresponded to women between the ages of 17 and 19, just over half were of urban origin, and less than half had a nuclear family. Regarding motivation, just under half entered the Instituto "Dr. Andrés Barbero" for personal reasons, opted for a career in Obstetrics for professional reasons and regarding the expectations of the students about their university training, personal reasons predominated.

Keywords: Expectations, students, university education.

\section{INTRODUCCIÓN}

Desde el ingreso a la universidad y durante toda la vida universitaria, cada estudiante formula diferentes ideas y creencias respecto a su formación profesional, la carrera que cursa, la institución elegida, y su futuro laboral. Las expectativas son creencias que se construyen con base en el entorno cambiante del estudiante. Estas en el ámbito académico, contemplan la elección de la carrera, el proceso de enseñanza-aprendizaje o participación en actividades de investigación. Esperan que la universidad les brinde una formación de calidad que les facilite su inserción laboral. En el ámbito extraacadémico incluyen actividades formativas extracurriculares, participación en talleres culturales o artísticos, asistencia, participación u organización de eventos académicos, práctica de deportes universitarios, fiestas estudiantiles ${ }^{(1)}$.

Dentro del ámbito de la educación superior, un área que atraviesa por una crisis de consolidación es la formación profesional de los estudiantes, que abarca no solo los perfiles de ingreso y egreso, sino todo aquello que se le proporciona al estudiante para su futuro desempeño profesional. Saber cuáles son las expectativas que presentan los estudiantes con respecto a su profesión y sí se relaciona con las expectativas que la universidad y la sociedad han destinado para ellos ${ }^{(2)}$.

Al momento de pensar en algún grado de intervención en la elección de lo que deparará el futuro de un estudiante que recién egresa de sus estudios secundarios, existen diversas hipótesis sobre cuáles son los factores que tienen más o menos incidencia sobre las decisiones. Existen dimensiones sobre las que se puede analizar la decisión de postular o no a una institución de educación superior, como ser: la naturaleza de los cursos, prestigio de la universidad, ansiedad del proceso de postulación y temor a fallar. No sólo, estar pensar en las ventajas económicas que podría obtener al tener un grado académico, sino también en las razones que motivan el ingreso a una institución de educación superior, necesidad de obtener un grado mayor de calificación, optar a mejores trabajos, perfeccionarse en nuevas áreas y disfrutar de la vida universitaria. Es importante considerar la influencia que pueden llegar a ejercer las redes sociales, familia, amigos como así también los profesores de la educación segundaria(3).

La mayoría de los estudiantes al llegar a estudios superiores, únicamente se esfuerzan cuando consideran que la materia que tienen que aprender resulta relevante, cognitivamente desafiante o profesionalmente "útil". La voluntad para aprender se relaciona con sus experiencias subjetivas, con su disposición y razones para involucrase en las actividades académicas ${ }^{(4)}$.

Así también, poseen percepciones relativas a su futuro, como la obtención del título profesional a corto plazo, continuación de sus estudios en segunda especialización, maestrías o diplomados. Aunado a su objetivo académico, también les interesa su futuro a mediano y largo plazo, que se resume en un mejor nivel de vida. Sin embargo, al finalizar los estudios se enfrentan con un mercado laboral donde no encuentran el trabajo deseado o los salarios no cumplen sus expectativas la proyección de vida puede verse afectada ${ }^{(5)}$.

El objetivo fue Determinar expectativas del estudiante sobre su formación universitaria. Carrera de Obstetricia. Instituto Dr. Andrés Barbero. San LorenzoParaguay. 2018. 


\section{MATERIALES Y MÉTODOS}

Diseño de estudio observacional, descriptivo, de corte transversal, enfoque cuantitativo. Se realizó en el Instituto Dr. Andrés Barbero, Carrera de Obstetricia (IAB - UNA), cuenta con 253 estudiantes matriculados del primer al quinto año de la carrera, el curriculum está diseñado por cursos, distribuidos en 10 semestres, abarca cuatro grandes áreas: Obstetricia, Neonatología, Salud Comunitaria y Ginecología, cuya duración es de 5 años, las clases teóricas se desarrollan en la casa central sito en San Lorenzo Departamento Central, las prácticas se realizan en las distintas dependencias del Ministerio de Salud Pública y Bienestar Social, además en el Hospital de Clínicas de la Facultad de Ciencias Médicas de la Universidad Nacional de Asunción. Cabe mencionar que cuenta con una filial en la ciudad de Concepción. Como requisito para optar al título de Licenciatura en Obstetricia es la presentación y defensa oral de la tesina. Población 253 estudiantes matriculados de la Carrera de Obstetricia casa Central. Muestra 25 estudiantes matriculados del primer curso. Muestreo censal. Tamaño de Muestra total de estudiantes del primer curso. Criterios de inclusión y exclusión estudiantes, del primer curso, de ambos sexos, de todas las edades, que acepten participar del estudio. El método fue la encuesta, la técnica la entrevista, e instrumento el cuestionario, es decir un formulario impreso con preguntas abiertas y cerradas, elaborado conforme a las variables de la investigación. Procedimiento para la recolección de los datos fue solicitar permiso a través de una nota firmada por la Directora General del Instituto Dr. Andrés Barbero, y por la Directora de la Carrera de Obstetricia, explicando la importancia y objetivos de la investigación, con el visto bueno, se solicitó la lista de estudiantes del primer curso, afín de contactar con los sujetos de estudio, para concretar fecha y hora para realizar el trabajo de campo, el cual no interfirió en el desarrollo normal de clases teóricas/prácticas, la encuesta fue auto-administrada, previo consentimiento informado verbal. Razón por la cual se estableció un cronograma de actividades, de común acuerdo. Los gastos de la investigación fueron solventados por la investigadora. Plan de tabulación y análisis de los datos se procedió a cargar los datos en una planilla electrónica Microsoft Excel, se depuró la base de datos, se exportó al programa EPIINFO 7.2 para el análisis. Se aplicó estadística descriptiva, se utilizaron estadísticos de tendencia central, dispersión, frecuencia absoluta y porcentajes, los resultados fueron representados en gráficos y tablas. Limitaciones del estudio fueron subsanadas durante el proceso de la investigación. En todo momento se consideraron los principios éticos.

\section{RESULTADOS}

Los datos sociodemográficos del estudiante, $92 \%$; corresponde al grupo de edad de 17 a 19 años; $88 \%$ del sexo femenino; $68 \%$ de procedencia urbana; $44 \%$ con quien vive familia nuclear.

Tabla 1: Frecuencia y porcentaje de estudiantes universitarios según datos sociodemográficos. Carrera de Obstetricia. San Lorenzo-Paraguay. 2018. $n=25$

\begin{tabular}{lcc}
\hline \multicolumn{1}{c}{ Datos sociodemográficos } & f & \% \\
\hline Grupo de edad (años) & $\mathbf{2 3}$ & $\mathbf{9 2}$ \\
$\mathbf{1 7}$ a 19 & 2 & 8 \\
20 a 22 & $\mathbf{2 2}$ & $\mathbf{8 8}$ \\
Sexo & 3 & 12 \\
$\begin{array}{l}\text { Femenino } \\
\text { Masculino }\end{array}$ & & \\
Procedencia & $\mathbf{1 7}$ & $\mathbf{6 8}$ \\
Urbano & 8 & 32 \\
Rural & & \\
Con quién vive & $\mathbf{1 1}$ & $\mathbf{4 4}$ \\
Nuclear & 7 & 24 \\
Monoparental & 7 & 24 \\
\hline
\end{tabular}

Fuente: Datos obtenidos por las autoras 
Motivos de estudiante para ingresar al Instituto Dr. Andrés Barbero, 40\% personal; 32\% Profesional; 20\% Institucional; 8\% Familiar.

Fuente: Datos obtenidos por las autoras

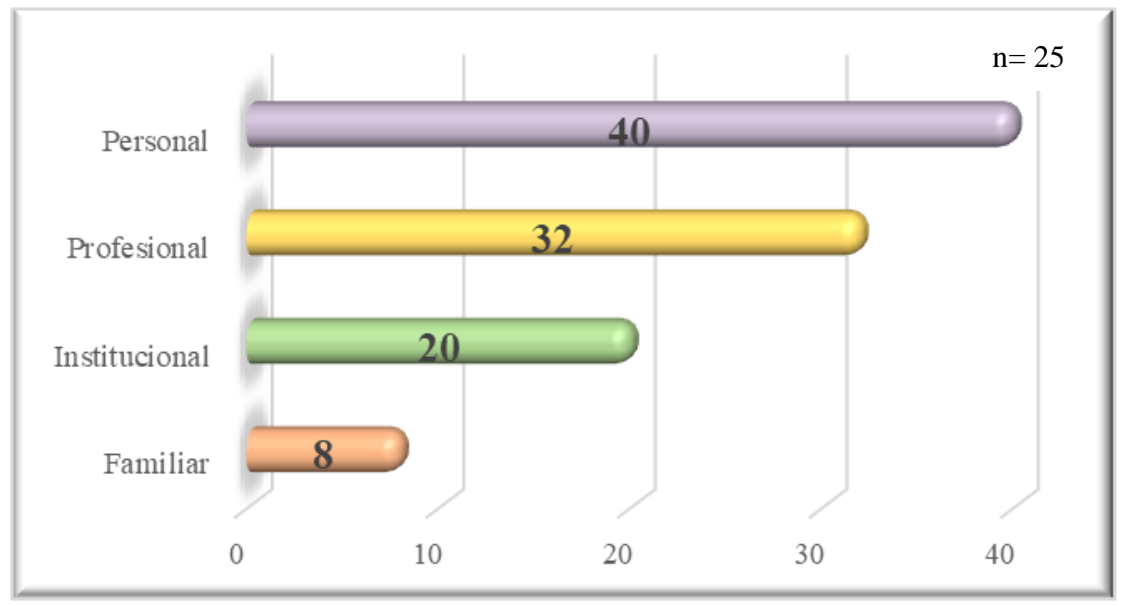

Figura 1: Distribución porcentual de estudiantes según motivos para ingresar al Instituto Dr. Andrés Barbero. Carrera de Obstetricia. San Lorenzo-Paraguay. 2018.

Motivos del estudiante para optar por la Carrera de Obstetricia, 36\% profesional; $20 \%$ personal; $16 \%$ humanitaria; $16 \%$ otros; $12 \%$ vocación, (Figura 2 ).

Fuente: Datos obtenidos por las autoras

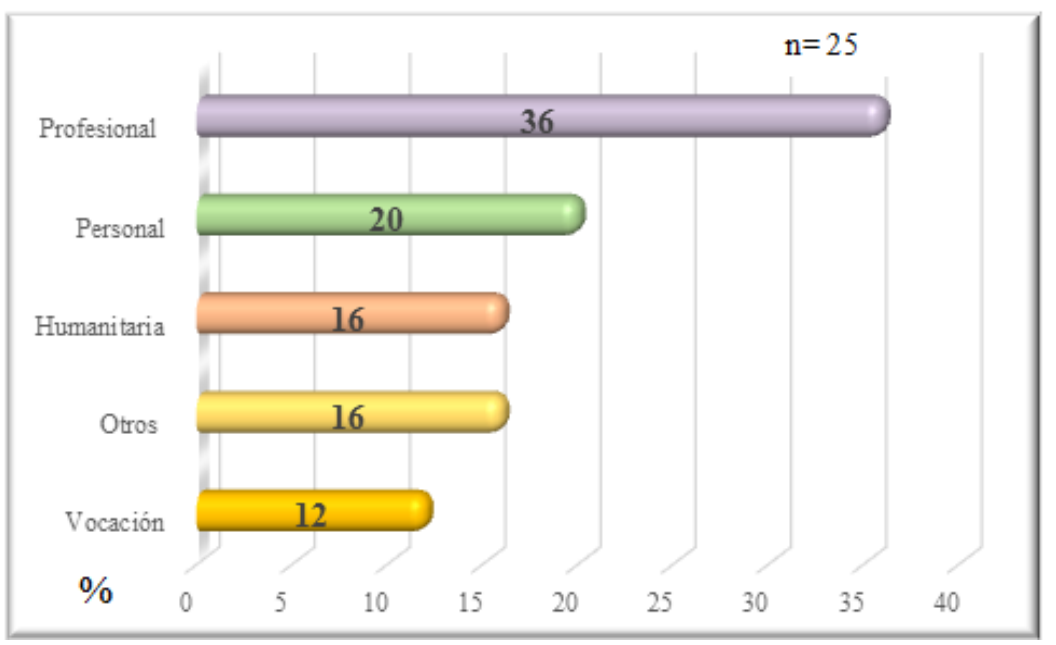

Figura 2: Distribución porcentual de estudiantes según expectativas para optar por la Carrera de Obstetricia. Carrera de Obstetricia. San Lorenzo-Paraguay. 2018.

Expectativas del estudiante sobre su formación universitaria en la Carrera de Obstetricia: personales $80 \%$ antes, $42 \%$ hoy, $66 \%$ futuro; institucional $12 \%$ antes, 36 $\%$ hoy; profesional $8 \%$ antes, $22 \%$ hoy, $34 \%$ futuro (Figura 3 ). 
Fuente: Datos obtenidos por las autoras

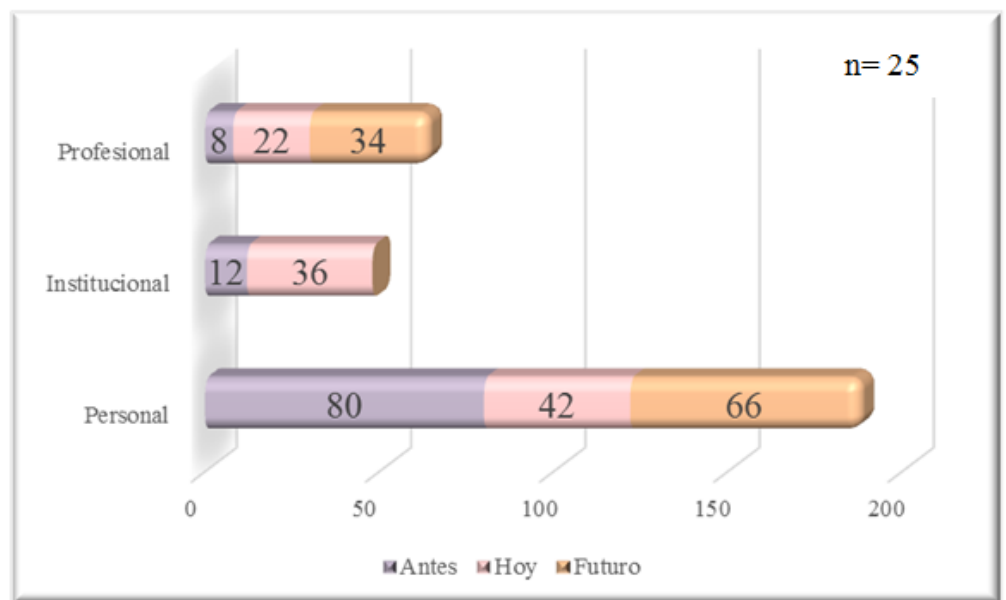

Figura 3: Distribución porcentual de estudiantes según expectativas sobre su formación universitaria en la carrera. Carrera de Obstetricia. IAB. UNA. San LorenzoParaguay. 2018

\section{DISCUSIóN}

La mayoría corresponde al grupo etario de 17 a 19 años, del sexo femenino, la totalidad estado civil soltero, procedencia del área urbana, viven en una familia nuclear. Sin embargo, en la Pontificia Universidad Católica de Argentina, los estudiantes contaban entre 17 y 21 años de edad al ingreso a la universidad ${ }^{(6)}$. En la Universidad de Santiago de Compostela $98,2 \%$ son mujeres matriculadas para estudios universitarios ${ }^{(7)}$. Estado civil la totalidad corresponde a estado de soltería de los participantes ${ }^{(8)}$. La mayor parte respondió que fueron por motivos personales $40 \%$, $32 \%$ profesional. Similar resultado, para los programas de posgrados de la Universidad Autónoma de México los estudiantes respondieron que su motivación intrínseca fue buscar su superación personal o simplemente tener un sentimiento de autorrealización; el segundo lugar está el conocimiento( ${ }^{(9)}$. Sin embargo, los estudiantes de psicología consideran como asunto personal, permite realizarse, "ser alguien en la vida"; así también como asunto familiar, puesto que el ingreso a la universidad es importante para la familia por varias razones como estatus, tener un hijo en la universidad, mejor condición económica, por la posibilidad de encontrar un buen empleo que permita ayudar a sus padres y hermanos ${ }^{(10)}$. Para los egresados de la Facultad de Economía, refieren los motivos por las cuales eligieron estudiar en la UNA, $47 \%$ por el prestigio de la Universidad Nacional de Asunción como principal razón ${ }^{(11)}$. El $36 \%$ del estudiante optó por la Carrera de Obstetricia por motivo profesional, seguido de lo personal, humanitaria, vocación. Sin embargo, los resultados obtenidos de la investigación realizada por Troncoso, Garay y Sanhueza difieren, los encuestados refieren que las motivaciones encontradas para la selección de su futura profesión se centran especialmente en las aspiraciones altruistas de los estudiantes, identificando la vocación de servicio público para contribuir a la sociedad $^{(12)}$. Estudiantes del primer curso de la Facultad de Medicina de la Universidad Complutense de Madrid (UCM), respondieron como motivo predominante $42 \%$ vocación, $24 \%$ interés científico, $14 \%$ altruista al servicio y entrega, $10 \%$ gratificación personal y $5 \%$ circunstancia personal ${ }^{(13)}$. En otra investigación, $38.1 \%$ de los estudiantes del centro Regional de Educación Normal "Dr. Gonzalo Aguirre Beltrán" aluden que el motivo para estudiar una carrera no es ni su vocación, ni el servir a la comunidad, sino "otros motivos", es decir se relacionan básicamente a factores de carácter económico o familiar ${ }^{(14)}$. En Bachilleres de colegios privados y públicos de la Cuidad Barrancabermeja, los alumnos manifestaron estar interesados a elegir una carrera universitaria por motivos personales un $42 \%$; generar beneficios a la sociedad $32 \%$; la posibilidad de optar con un buen salario $22 \%$ y por último la influencia familiar $4 \%{ }^{(15)}$. Los resultados coinciden con Boza y Toscano quienes estudió los 
motivos de elección de la carrera a estudiantes de 12 universidades españolas, donde los motivos de carácter profesional (salidas profesionales y deseo de lograr un trabajo estable y bien remunerado), carácter personal (valor formativo de los estudios, búsqueda de autorrealización personal) ${ }^{(16)}$. Las expectativas del estudiante sobre su formación universitaria en la Carrera de Obstetricia: Personal $80 \%$ antes, $42 \%$ hoy, $66 \%$ futuro; Institucional $12 \%$ antes, $36 \%$ hoy; Profesional $8 \%$ antes, $22 \%$ hoy, $34 \%$ futuro. Los resultados de Carrasco y Colaboradores difieren, los estudiantes relevan que los obstáculos institucionales son: falta de orientación vocacional, subvaloración del potencial de los estudiantes, falencias en la formación académica, elementos que afectan tanto la toma de decisión vocacional, como el posterior rendimiento académico en la universidad ${ }^{(17)}$. Orozco describe en su indagación que los estudiantes buscan una universidad que tenga buen prestigio, buena infraestructura, bien posicionada en los rankings, profesores accesibles y cercanos, ambiente universitario agradable ${ }^{(3)}$.

La investigación realizada tuvo como objetivo general determinar la expectativa de estudiantes sobre su formación universitaria, la mayoría de los estudiantes corresponden al grupo de edad de 17 a 19 años, sexo femenino, procedencia urbana, viven en el seno de una familia nuclear; poco menos de la mitad ingreso al Instituto Dr. Andrés Barbero por razones personales; optó por la carrera de Obstetricia por motivo profesional; expectativas sobre su formación universitaria predomina las razones personales.

\section{REFERENCIAS BIBLIOGRÁFICAS}

1. Cuamea Lizárraga GM, Mondaca Cota A. Expectativas y realidades de estudiantes universitarios: la relación profesor-alumno. Congreso Internacional de Investigación ISSN 1946-5351 Online 1948-2353 CD ROM. 5(3) pp.755-760. México. 2013.

2. Hernández G, Fernández J. Expectativas Profesionales: Un Estudio de Caso. Rev Mex Orientación Educ [Internet]. 2010; 7(19):18-27. Available from: http://pepsic.bvsalud.org/scielo.php?scr ipt $=$ sci_arttext\&pid $=$ S1665-

$75272010000200004 \&$ Ing $=$ pt\&nrm $=$ iso \&tlng $=\mathrm{es}$

3. Orozco AL, Manzur E. Variables que inciden en la elección de carrera y casa de estudios, en la perspectiva del género. 2009; 4-82.

4. Steinman A, Bosch B, Aiassa D. Motivación y Expectativas de los estudiantes para aprender ciencias en la Universidad. Motiv to Learn From theory to Pract [Internet]. 2013; 3(57):58598. Available from: http://www.redalyc.org/articulo.oa?id= 14025774012

5. Tarqui Mamani CB, Sanabria Rojas HA, Zárate Cárdenas E. Expectativas de laborar en el primer nivel de atención de salud de los estudiantes de una facultad de medicina de Lima, Perú. An la Fac Med. 2015; 76(1):57.

6. Castillo MAC. Universidad de la República Oriental del Uruguay Facultad de Psicología. 2016; 20. Available from: https://sifp.psico.edu.uy/sites/default/fi les/Trabajos finales/

Archivos/manuel_cavia_pre_proyecto_de_investigacion.pdf

7. Porto Castro AM, Mosteiro García MJ. Los Motivos de elección de estudios de las estudiantes universitarias: un análisis en función del tipo de Carrera. Universidad de Santiago de Compostela. 2003. Disponible en: http://www.fessociologia.com/files/congress/10/grupos -trabajo/ponencias/513.pdf.

8. Vega Barrera JL. Influencia de los padres en la elección de carrera desde la Perspectiva del estudiante universitario. 2003; 88. Available from: http://cdigital.dgb.uanl.mx/te/1020149 303.PDF

9. Alcocer LAE, Rojas CA. Motivos de estudiantes de nuevo ingreso para estudiar un posgrado en educación. Rev Iberoam Educ. 1999;1-15.

10. Villamizar Acevedo GA, Delgado Meza JA. Identificación de los motivos para estudiar psicología en estudiantes de primer año de la Corporación Universitaria de Investigación y Desarrollo -UDI. Espacios. 2017; 38(30).

11. Miranda Espinoza MA. Egresados de la facultad de ciencias económicas (una), y su incorporación al mercado laboral del sector público de la administración central período: 2003 - 2013. Paraguay. 2016. Disponible en https://www.eco.una.py/eco/postgrado/ tesis/2016/Maria_de_los_Angeles_Miran da_espinoza_110416.pdf

12. Pantoja CT, Lara BG, Muñoz PS. Percepción de las motivaciones en el ingreso a una carrera del área de la 
salud. Horiz Med (Barcelona). 2016; 16(1):55-61.

13. Gutierrez S, Cuenca D, Alvarez O. ¿Por qué quiero ser médico? Educ Médica [Internet]. 2008; 11(Supl 1):1-6. Available from: http://scielo.isciii.es/scielo.php?pid=S1 575-

$18132008000500001 \&$ script=sci_arttex t\&tlng $=p t$

14. Said-Hung E, Gratacós G, Cobos JV. Factores que influyen en la elección de las carreras de pedagogía en Colombia. Educ e Pesqui. 2017; 43(1):31-48.

15. Bravo Torres G, Vergara Tamayo $M$. Factores que determinan la elección de carrera profesional: en estudiantes de undécimo grado de colegios públicos y privados de Barrancabermeja. Psicoespacios Rev virtual la Inst Univ Envigado. 2018; 12(20):35-48.
16. Boza Carreño A, Toscano Cruz M de la O. Motivos, Actitudes y Estrategias de Aprendizaje: Aprendizaje Motivado en Alumnos Universitarios. Profesorado. Revista de Curriculum y Formación del Profesorado Vol. 16, No 1 (enero-abril 2012) ISSN 1138-414X (edición papel) ISSN 1989-639X (edición electrónica) Fecha de recepción 22/10/2011 Fecha de aceptación 06/04/2012. Disponible en:

http://www.redalyc.org/articulo.oa?id= 56724377008. 2012.

17. Carrasco E, Zúñiga C, Espinoza J. Elección de Carrera en Estudiantes de Nivel Socioeconómico Bajo de Universidades Chilenas Altamente Selectivas. Calidad en la Educación $n^{\circ}$ 40, julio 2014.2019 (18 de febrero de 2019) pg. 96-128. 